\title{
EDGE ASYMPTOTICS ON A SKEW CYLINDER: COMPLEX VARIABLE FORM
}

\author{
MARTIN COSTABEL \\ CeReMaB, Université de Bordeaux 1 \\ 351 cours de la Libération, F-33405 Talence Cedex, France \\ MONIQUE DAUGE \\ Département de Mathématiques, Université de Nantes \\ 2, rue de la Houssinière, F-44072 Nantes Cedex 03, France
}

\section{Position of the problem}

1.a. Introduction. It is well known that singularities of the domain give rise to a loss of regularity for the solutions of any elliptic boundary value problem. The situation is rather well understood when the singularities are isolated points of the boundary and are of conical type (see [5], [9]).

When a conical singularity is tensorized with an affine space, one gets an edge. Regularity results are rather complete in that case [8], [13]. If the operator is translation invariant along the edge, the asymptotics can be derived in a direct way from the asymptotics on the corresponding conical domain [3].

But for physical examples in the ordinary three-dimensional space, when a bounded domain has edges and no corners, then the edges are necessarily curved. The simplest example is a cylinder with circular basis, cut orthogonally to its generating lines. But this example is very particular: The opening of the edge is everywhere $\pi / 2$ and the curvature of the edge is constant. If one cuts the cylinder by a plane which is skew with respect to the generating lines, then the edge is elliptic and the opening angle is varying. This gives rise to difficulties for the precise analysis of the structure of the solution, due to the fact that the asymptotics in the corresponding two-dimensional domains depend in a discontinuous way on the opening parameter. In particular, the coefficients of the singular functions along the edge (stress intensity factors etc.) will blow up at certain points. Such a behavior causes difficulties also for numerical approximations. 
In this note, we consider as model problem the mixed Dirichlet-Neumann problem for the Laplace operator on a skew cylinder. This problem was posed as a question to the first author by I. Babuška (Maryland). We already stated results for this problem in [1]. Here we present improved results. With the introduction of a complex variable $\zeta$ in the normal plane to the edge, we give expressions of the edge asymptotics in a simplified form. Such a form is based upon the divided differences of the function $\lambda \mapsto \zeta^{\lambda}$ calculated at the exponents of singularities.

This formulation was inspired by a recent paper by Maz'ya and Rossmann [11] where a different but related problem was treated, namely the problem of writing the corner singularities of a two-dimensional Dirichlet problem for the Laplacian in a form that is stable with respect to variations of the corner angle. It is in fact not hard to see that our formulation is equivalent to that given by Maz'ya and Rossmann, if we consider the edge angle $\omega(y)$ as independent unknown instead of the edge variable $y$. The possibility of using powers of a complex variable to describe singularities in piecewise analytic plane domains was shown in the earlier work [7].

The restriction to the case of the Laplace operator is actually not as serious a limitation as it may look: The results we state here can be extended to any strongly elliptic boundary value problem for a second order operator with real analytic coefficients.

1.b. Boundary value problem. Let $B$ be an analytic bounded domain in $\mathbb{R}^{2}$. Let $\Psi$ be an affine function $\left(x_{2}, x_{3}\right) \mapsto x_{1}=\Psi\left(x_{2}, x_{3}\right)$. We assume that

$$
\Psi\left(x_{2}, x_{3}\right)>0 \quad \text { for all }\left(x_{2}, x_{3}\right) \in \bar{B} \text {. }
$$

We introduce

$$
\Omega=\left\{\left(x_{1}, x_{2}, x_{3}\right) \in \mathbb{R}^{3} \mid\left(x_{2}, x_{3}\right) \in B, 0<x_{1}<\Psi\left(x_{2}, x_{3}\right)\right\} .
$$

This is our skew cylinder. We denote by $M$ the top edge and by $\partial_{1} \Omega$ the side of the cylinder:

$$
\partial_{1} \Omega=\left\{\left(x_{1}, x_{2}, x_{3}\right) \in \mathbb{R}^{3} \mid\left(x_{2}, x_{3}\right) \in \partial B, 0<x_{1}<\Psi\left(x_{2}, x_{3}\right)\right\} .
$$

The union of the top and the bottom of the cylinder is denoted by $\partial_{2} \Omega$.

The boundary value problem that we choose to consider here is the following:

$$
\left(\mathcal{P}_{\Omega}\right) \quad\left\{\begin{array}{l}
\Delta u=f \quad \text { on } \Omega, \\
u=0 \quad \text { on } \partial_{1} \Omega, \quad \partial u / \partial n=h \quad \text { on } \partial_{2} \Omega .
\end{array}\right.
$$

This example is interesting because the singularities appear at a low regularity level: in a generic way, $u \notin H^{2}(\Omega)$.

1.c. Singularities on plane sectors. Let $\omega \in(0,2 \pi)$ and let $G(\omega)$ denote the two-dimensional angle with opening $\omega$. We use polar coordinates $(r, \theta)$ so that $G(\omega)$ corresponds to $r>0,0<\theta<\omega$. 
The boundary value problem corresponding to $\left(\mathcal{P}_{\Omega}\right)$ is:

$\left(\mathcal{P}_{G}\right) \quad\left\{\begin{array}{l}\Delta w=\widetilde{f} \quad \text { on } G, \\ w=0 \quad \text { on } \theta=0, \quad \partial w / \partial n=\widetilde{h} \quad \text { on } \theta=\omega .\end{array}\right.$

For any integer $k \geq 1$, we set

$$
\nu_{k}=(2 k-1) \frac{\pi}{2 \omega}
$$

It is well known [4] that the singularities of any solution of $\left(\mathcal{P}_{G}\right)$ are linear combinations of the functions $\sigma_{k}$ (when $\nu_{k} \notin \mathbb{N}$ ) and $\tau_{k}$ (when $\nu_{k} \in \mathbb{N}$ ) below:

$$
\begin{aligned}
\sigma_{k}(r, \theta) & =r^{\nu_{k}} \sin \nu_{k} \theta, \\
\tau_{k}(r, \theta) & =r^{\nu_{k}}\left(\log r \sin \nu_{k} \theta+\theta \cos \nu_{k} \theta\right) .
\end{aligned}
$$

Let us write the plane coordinates in complex form: $\zeta=r e^{i \theta}$. Then we have

$$
\sigma_{k}(r, \theta)=\operatorname{Im} \zeta^{\nu_{k}}, \quad \tau_{k}(r, \theta)=\operatorname{Im} \zeta^{\nu_{k}} \log \zeta .
$$

Proposition 1.1. Let $s \in \mathbb{R}, s>1 / 2$. Let $w$ be a solution of $\left(\mathcal{P}_{G}\right)$ such that $\tilde{f}$ has $H^{s-1}$ regularity and that $\widetilde{h}$ has $H^{s-1 / 2}$ regularity. Assume that $w$ has compact support. Then $w$ admits the decomposition

$$
w=w_{\text {reg }}+w_{\text {sing }} \quad \text { with } \quad w_{\text {reg }} \in H^{s+1-\varepsilon}(G) \forall \varepsilon>0
$$

and

$$
w_{\text {sing }}=\sum_{\nu_{k}<s, \nu_{k} \notin \mathbb{N}} c_{k} \sigma_{k}+\sum_{\nu_{k}<s, \nu_{k} \in \mathbb{N}} c_{k} \tau_{k} .
$$

1.d. Aims. Let us return now to our skew cylinder. We assume that for a fixed real $s>1 / 2$

$$
f \in H^{s-1}(\Omega), \quad h \in H^{s-1 / 2}\left(\partial_{1} \Omega\right) .
$$

Then there exists a variational solution $u \in H^{1}(\Omega)$.

For any $y \in M$, let $\omega(y)$ be the opening angle of $\Omega$ at $y$. We set $\nu_{1}(y)=$ $\pi /(2 \omega(y))$. If $s<\min _{y \in M} \nu_{1}(y)$, then $u \in H^{s+1}(\Omega)$. From now on we assume that

$$
\min _{y \in M} \nu_{1}(y)<s
$$

Then $u$ can be split into two parts for any $\varepsilon>0$,

$$
u=u_{\text {reg }}+u_{\text {sing }}
$$

where $u_{\text {reg }} \in H^{s+1-\varepsilon}(\Omega)$ and $u_{\text {sing }}$ is an asymptotics. Our aim is to describe the structure of such splittings. In particular, we want to separate as much as possible the roles of the different variables, the abscissa $y$ along the edge, the distance $r$ from the edge and the angular variable $\theta$. More precisely, we intend to separate what comes from the geometrical framework (domain and boundary value problem) and what comes from the data $(f, h)$.

1. The part that comes from the geometrical framework will be described as special combinations of real and imaginary parts of powers of $\zeta$ and $\bar{\zeta}$. The 
introduction of such combinations allows us to get rid of the discontinuity in the expression of the singularities when the function $y \mapsto \nu_{k}(y):=(2 k-1) \pi /(2 \omega(y))$ crosses an integer.

2. The part that comes from the data will be described by some coefficients $c(y)$ along the edge. The regularity of $c$ depends on $s$ and on the exponents of the associated singularity.

\section{Simple asymptotics}

2.a. Simple singularities in two-dimensional domains. Recall that for each $y$ in the edge $M, \omega(y)$ denotes the opening of $\Omega$ at $y$. We can choose local cylindrical coordinates $(y, r, \theta)$ such that $r=0$ corresponds to the edge and such that $0<\theta<\omega(y)$ describes locally the domain $\Omega$.

What can be expected as singularities along the edge for the solution of prob$\operatorname{lem}\left(\mathcal{P}_{\Omega}\right)$ is

$$
\sum_{\alpha} c_{\alpha}(y) S_{\alpha}(y, r, \theta)
$$

where the functions $S_{\alpha}$ do not depend on the data $(f, h)$.

First, we want to link the form of the $S_{\alpha}(y, \cdot, \cdot)$ with the form of singularities of some two-dimensional boundary value problems on $G(\omega(y))$. Due to the presence of curvature terms, it is natural to consider instead of $\Delta$ as interior operator on $G$ a more general second order operator

$$
A\left(z ; \partial_{z}\right)=\sum_{|\beta| \leq 2} a_{\beta}(z) \partial_{z}^{\beta} \quad \text { with } a_{\beta} \in C^{\infty}(\bar{G}) \text { and } a_{\beta}(0)=\delta_{\beta} .
$$

This means that the principal part of $A$ at the vertex 0 is $\Delta$.

Secondly, we want the functions $S_{\alpha}$ to depend smoothly on $y$. In a first stage we search them as powers of $\zeta$ and $\bar{\zeta}$. That is why we will assume that $\nu_{k} \notin \mathbb{N}$ to avoid the discontinuity between $\sigma_{k}$ and $\tau_{k}$.

Proposition 2.1. Let $A$ be an elliptic operator satisfying (2.2). Let $\omega \in$ $(0,2 \pi)$. We assume that

$$
\forall k \geq 1 \text { such that } k \pi / \omega<2 s, \quad k \pi / \omega \notin \mathbb{N} .
$$

Let $w$ be a solution of problem $\left(\mathcal{P}_{G(\omega)}\right)$ with $\Delta$ replaced by $A$ and satisfying the same hypotheses as in Proposition 1.1. Then $w$ can be split in the same way with

$$
w_{\text {sing }}=\sum_{k, l: \nu_{k}+l<s} \sum_{n=0}^{2 l} c_{k l n} \operatorname{Re}\left(e^{-2 i n \theta} \zeta^{\nu_{k}+l}\right)+c_{k l n}^{\prime} \operatorname{Im}\left(e^{-2 i n \theta} \zeta^{\nu_{k}+l}\right) .
$$

2.b. Simple asymptotics along the edge. The exponents of the singularities which appear in the asymptotics along the edge depend on the edge parameter $y$. They are the same as in two-dimensional problems as considered above in 
Proposition 2.1 in an angle with opening $\omega(y)$. We can enumerate them using a double index $\kappa=(k, l)$, where $k \in \mathbb{N}^{*}$ and $l \in \mathbb{N}$ :

$$
\nu_{\kappa}(y)=(2 k-1) \frac{\pi}{2 \omega(y)}+l \text {. }
$$

We could think that if we assume condition (2.3) for any $y$, then the functions $S_{\alpha}$ will be the real and imaginary parts of

$$
e^{-2 i n \theta} \zeta^{\nu_{\kappa}(y)}
$$

Indeed, the tangential derivatives $\partial_{y}$ produce logarithmic terms and the $S_{\alpha}$ are the real and imaginary parts of

$$
e^{-2 i n \theta} \zeta^{\nu_{\kappa}(y)} \log ^{q} \zeta
$$

We have also to note that an asymptotics in tensor product form such as (2.1) is not convenient in general, since the $c_{\alpha}$ are not regular enough. Therefore we define the usual (see [6], [3], [10]) regular extension of the coefficients: we introduce a function $\Phi(y, r)$ such that its partial Fourier transform satisfies

$$
\mathcal{F}_{y \rightarrow \xi} \Phi(\xi, r)=\varphi(r|\xi|)
$$

where $\varphi$ is a rapidly decreasing function, has a Fourier transform with compact support, and satisfies for a sufficiently large $N$

$$
\varphi(0)=1, \quad \frac{d^{n}}{d t^{n}} \varphi(0)=0 \quad(n=1, \ldots, N) .
$$

We define the convolution with respect to $y$,

$$
(c * \Phi)(y, r):=\int \Phi\left(y-y^{\prime}, r\right) c\left(y^{\prime}\right) d y^{\prime} .
$$

The following theorem describes our result on the "simple" edge asymptotics in complex variable form, i.e. when any crossing between exponents $\nu_{\kappa}$ themselves and with integer numbers is excluded.

THEOREM 2.2. Let $I, I^{\prime}$ be intervals such that the cylindrical local coordinates $(y, r, \theta)$ are defined in a neighborhood $\mathcal{U}$ of $I^{\prime}$ in $\Omega$ and $I \Subset I^{\prime}$. We suppose there is no crossing point in $I^{\prime}$ :

$$
\forall y \in I^{\prime}, \forall k \geq 1 \text { such that } k \pi / \omega(y)<2 s, \quad k \pi / \omega(y) \notin \mathbb{N},
$$

and that the $\nu_{\kappa}$ do not cross the value $s$ above $I^{\prime}$. Then any solution $u$ of problem $\left(\mathcal{P}_{\Omega}\right)$ with $f \in H^{s-1}(\Omega), h \in H^{s-1 / 2}\left(\partial_{1} \Omega\right)$ can be decomposed into

$$
u=u_{\text {reg }}+u_{\text {sing }} \quad \text { with } \quad u_{\text {reg }} \in H^{s+1-\varepsilon}(\mathcal{U}) \forall \varepsilon>0
$$

and

$$
\begin{aligned}
u_{\text {sing }}= & \sum_{\kappa, q, n}\left(c_{\kappa, q, n} * \Phi\right)(y, r) \operatorname{Re}\left(e^{-2 i n \theta} \zeta^{\nu_{\kappa}(y)} \log ^{q} \zeta\right) \\
& +\left(c_{\kappa, q, n}^{\prime} * \Phi\right)(y, r) \operatorname{Im}\left(e^{-2 i n \theta} \zeta^{\nu_{\kappa}(y)} \log ^{q} \zeta\right)
\end{aligned}
$$


The coefficients $c_{\kappa, q, n}(y)$ and $c_{\kappa, q, n}^{\prime}(y)$ are defined on $I$ and belong to $H^{s-\nu_{\kappa}}(y)-\varepsilon(I)$ for all $\varepsilon>0$. The sum extends over those $\kappa$ for which $\operatorname{Re} \nu_{\kappa}<s$ holds on $I$ and over $0 \leq n \leq 2 l$ if $\kappa=(k, l)$.

Simple asymptotics in "real variable form" involve instead of the real and imaginary parts of $e^{-2 i n \theta} \zeta^{\nu_{\kappa(y)}} \log ^{q} \zeta$ functions of the type $\varphi_{\kappa, q, n}(y, \theta) r^{\nu_{\kappa}(y)} \log ^{q} r$ where the functions $\varphi_{\kappa, q, n}(y, \theta)$ are analytic in all their arguments but only implicitly known. Such asymptotics are given in [6], [12], [10] and [1].

2.c. The crossing of exponents. Now we have to include all positive integers in the set of exponents due to the possible interaction between polynomials and singularities. That is the reason for introducing

$$
\nu_{\kappa}=l \quad \text { for } \kappa=(0, l) \text {. }
$$

The above assumption (2.6) implies that there is no crossing of exponents, i.e., there are no points $y$ such that $\nu_{\kappa}(y)=\nu_{\kappa^{\prime}}(y)$ for some $\kappa, \kappa^{\prime}$ with $\kappa \neq \kappa^{\prime}$. In $[6],[12],[10]$ such a condition is also required.

For our problem of the skew cylinder, it is impossible to avoid such crossings. For $y_{0}$ such that $\omega\left(y_{0}\right)=\pi / 2$ (there always exist two such points), we have

$$
\nu_{1,0}\left(y_{0}\right)=\nu_{0,1}\left(y_{0}\right)=1 \text {. }
$$

The points where crossing of exponents will eventually appear (for large $s$ ) are dense in $M$, so this phenomenon occurs in a generic way.

In Section 3, we will present the main results of this note for the case when crossing points are present. Our motivations for their presentation are the following.

1. To give an asymptotics in the neighborhood of crossing points which is as explicit and as simple as possible.

2. To eliminate as many technical hypotheses as possible.

\section{Asymptotics at crossing points}

3.a. Ordering the exponents. Let $y_{0}$ be a crossing point, i.e., a point where there exist distinct $\kappa$ and $\kappa^{\prime}$ such that

$$
\nu_{\kappa}\left(y_{0}\right)=\nu_{\kappa^{\prime}}\left(y_{0}\right)<s .
$$

Since we assume that our cylinder is actually skew, crossing points are isolated, so there exist open intervals $I$ and $I^{\prime}$ with $y_{0} \in I, \bar{I} \subset I^{\prime}$, and there is no other crossing point in $\overline{I^{\prime}}$.

Let $\mathcal{K}_{y_{0}}$ be the set of indices,

$$
\mathcal{K}_{y_{0}}:=\left\{\kappa=(k, l) \in \mathbb{N}^{2} \mid \nu_{\kappa}\left(y_{0}\right)<s\right\} .
$$

We denote by $\mu_{1}^{0}, \ldots, \mu_{j_{0}}^{0}$ the distinct elements of the set

$$
\left\{\nu_{\kappa}\left(y_{0}\right) \mid \kappa \in \mathcal{K}_{y_{0}}\right\} .
$$


Since $y_{0}$ is a crossing point, the cardinality of $\mathcal{K}_{y_{0}}$ is strictly larger than $j_{0}$. For each $j$, let $\mathcal{K}_{y_{0}, j}$ be the subset of $\mathcal{K}_{y_{0}}$,

$$
\mathcal{K}_{y_{0}, j}:=\left\{\kappa \in \mathcal{K}_{y_{0}} \mid \nu_{\kappa}\left(y_{0}\right)=\mu_{j}^{0}\right\} .
$$

The $\mu_{j}^{0}$ are either crossing exponents (if $\# \mathcal{K}_{y_{0}, j}>1$ ) or simple exponents (if $\left.\# \mathcal{K}_{y_{0}, j}=1\right)$.

For each $\kappa$, the multiplicity of $\nu_{\kappa}$ is the maximal power of $\log \zeta$ which appears in the asymptotics (2.8) along with the term $\zeta^{\nu_{\kappa}(y)}$ for $y \in I \backslash\left\{y_{0}\right\}$. Then we denote by $\left(\kappa_{j}^{q}\right)_{1 \leq q \leq q_{j}}$ an enumeration of $\mathcal{K}_{y_{0}, j}$, repeating each term according to its multiplicity.

Finally, we set for $y \in I^{\prime}$ :

$$
\underline{\nu}_{j}(y):=\max _{\kappa \in \mathcal{K}_{y_{0}, j}} \nu_{\kappa}(y) .
$$

3.b. Divided differences. What essentially changes from the simple asymptotics (2.8) is the behavior of the functions of $\zeta$. Instead of having separately the terms $\zeta^{\nu_{\kappa}(y)} \log ^{q} \zeta$, we have now special combinations of these terms which cannot be separated, namely the divided differences of the function $\lambda \mapsto \zeta^{\lambda}$ at some of the points $\nu_{\kappa_{1}}(y), \ldots, \nu_{\kappa_{q}}(y)$ for each $y \in I$. Recall that, when $\mu_{1}, \ldots, \mu_{K}$ are all distinct, the divided difference of the function $w$ at the $K$-tuple $\mu_{1}, \ldots, \mu_{K}$ is defined by the classical recursion formula:

$$
w\left[\mu_{1}\right]=w\left(\mu_{1}\right)
$$

and for $l=2, \ldots, K$

$$
w\left[\mu_{1}, \ldots, \mu_{l}\right]=\frac{1}{\mu_{1}-\mu_{l}}\left(w\left[\mu_{1}, \ldots, \mu_{l-1}\right]-w\left[\mu_{2}, \ldots, \mu_{l}\right]\right) .
$$

Moreover, for analytic functions $w$ one has for any $\mu_{1}, \ldots, \mu_{K}$ not necessarily distinct

$$
w\left[\mu_{1}, \ldots, \mu_{K}\right]=\frac{1}{2 i \pi} \int_{\gamma} \frac{w(\lambda)}{\prod_{l=1}^{K}\left(\lambda-\mu_{l}\right)} d \lambda
$$

where $\gamma$ is a simple curve surrounding all $\mu_{l}$. Thus, for any $\zeta$ we consider

$$
S\left[\mu_{1}, \ldots, \mu_{K} ; \zeta\right]=\frac{1}{2 i \pi} \int_{\gamma} \frac{\zeta^{\lambda}}{\prod_{l=1}^{K}\left(\lambda-\mu_{l}\right)} d \lambda .
$$

We see that $S\left[\mu_{1}(y), \ldots, \mu_{K}(y) ; \zeta\right]$ with analytic $\mu_{1}(y), \ldots, \mu_{K}(y)$ is a linear combination of terms of the form $\zeta^{\mu_{l}(y)} \log ^{q} \zeta$ with coefficients that are meromorphic in $y$. If all $\mu_{l}(y)$ are equal to the same $\mu(y)$ then

$$
S[\underbrace{\mu, \ldots, \mu}_{q+1 \text { times }} ; r]=\frac{1}{q !} r^{\mu} \log ^{q} r
$$


When all the $\mu_{l}(y)$ are distinct, we obtain

$$
S\left[\mu_{1}, \ldots, \mu_{K} ; \zeta\right]=\sum_{l=1}^{K} \frac{\zeta^{\mu_{l}}}{\prod_{k=1, k \neq l}^{q}\left(\mu_{l}-\mu_{k}\right)} .
$$

THEOREM 3.1. Let $I, I^{\prime}$ be intervals satisfying the same assumption as in Theorem 2.2 except that hypothesis (2.6) is replaced by

$$
y_{0} \in I \text { is the only crossing point in } I^{\prime} \text {. }
$$

Then any solution $u$ of problem $\left(\mathcal{P}_{\Omega}\right)$ with $f \in H^{s-1}(\Omega), h \in H^{s-1 / 2}\left(\partial_{1} \Omega\right)$ can be decomposed into

$$
u=u_{\text {reg }}+u_{\text {sing }} \quad \text { with } \quad u_{\text {reg }} \in H^{s+1-\varepsilon}(\mathcal{U}) \forall \varepsilon>0
$$

and

$$
\begin{aligned}
u_{\text {sing }}= & \sum_{j, q, n}\left(d_{j, q, n} * \Phi\right)(y, r) \operatorname{Re}\left(e^{-2 i n \theta} S\left[\nu_{\kappa_{j}^{1}}(y), \ldots, \nu_{\kappa_{j}^{q}}(y) ; \zeta\right]\right) \\
& +\left(d_{j, q, n}^{\prime} * \Phi\right)(y, r) \operatorname{Im}\left(e^{-2 i n \theta} S\left[\nu_{\kappa_{j}^{1}}(y), \ldots, \nu_{\kappa_{j}^{q}}(y) ; \zeta\right]\right) .
\end{aligned}
$$

The coefficients $d_{j, q, n}(y)$ and $d_{j, q, n}^{\prime}(y)$ are defined on $I$ and belong to $H^{s-\underline{\nu}_{j}-\varepsilon}(I)$ for all $\varepsilon>0$. The index $n$ runs through $\left\{0,1, \ldots, 2 l_{j}\right\}$ where

$$
l_{j}:=\max \left\{l \in \mathbb{N} \mid \exists k \in \mathbb{N}: \nu_{k l}\left(y_{0}\right)=\mu_{j}^{0}\right\} .
$$

R e m a r k 3.2. If there is no crossing in $I^{\prime}$, then this statement yields the same result as Theorem 2.2. Indeed, the sets $\mathcal{K}_{y_{0}, j}$ are all reduced to one element and the functions in $\zeta$ are all of the form $S[\nu, \ldots, \nu ; \zeta]$, i.e. $\zeta^{\nu} \log ^{q} \zeta$ according to (3.5).

Rem ark 3.3. If $y$ and the multiplicities in (3.9) are such that all $\nu_{\kappa_{1}}, \ldots, \nu_{\kappa_{q}}$ are different, then one can write the singular function in (3.9) as

$$
e^{-2 i n \theta} S\left[\nu_{\kappa_{1}}(y), \ldots, \nu_{\kappa_{q}}(y) ; \zeta\right]=\bar{\zeta}^{n} \zeta^{-n} \sum_{l=1}^{q} a_{l}(y) \zeta^{\nu_{\kappa_{l}}(y)}
$$

with the coefficients

$$
a_{l}(y)=\prod_{\substack{r=1 \\ r \neq l}}^{q} \frac{1}{\nu_{\kappa_{l}}(y)-\nu_{\kappa_{r}}(y)} .
$$

3.c. The first singularity. Let us illustrate our statements by considerations about the first singularity of our problem $\left(\mathcal{P}_{\Omega}\right)$. We take $s>1$ and consider those points $y_{0}$ and $y_{0}^{\prime}$ such that $\omega\left(y_{0}\right), \omega\left(y_{0}^{\prime}\right)=\pi / 2$. Since there is one zero Dirichlet condition, $\nu_{(0,0)}$ does not appear. With that choice of $s$, the first exponents in the neighborhood of $y_{0}$ are $\nu_{(1,0)}$ and $\nu_{(0,1)}$ and they cross each other in $y_{0}$, and the same holds for $y_{0}^{\prime}$. For simplicity suppose that $s<2$ so that only $\nu_{(1,0)}$ and $\nu_{(0,1)}$ are relevant, and set

$$
\nu_{1}(y):=\nu_{(1,0)}(y)=\frac{\pi}{2 \omega(y)} \quad \nu_{2}(y):=\nu_{(0,1)}(y)=1
$$


For $y \neq y_{0}$ and $y$ in a neighborhood of $y_{0}$, the simple asymptotics (2.8) holds. Indeed, the terms involved in $u_{\text {sing }}$ are only the imaginary parts of $\zeta^{\nu_{j}}$ and the only contribution of $n$ is $n=0$ : The "simple asymptotics" of $u$ is

$$
c_{1} * \Phi \operatorname{Im} \zeta^{\nu_{1}}+c_{2} * \Phi \operatorname{Im} \zeta^{\nu_{2}} .
$$

The function $\operatorname{Im} \zeta^{\nu_{1}}$ corresponds to the first corner singularity and $\operatorname{Im} \zeta^{\nu_{2}}$ is a polynomial. Here it is possible to compute $c_{2}(y)$ since it depends only on the pointwise value of the Neumann boundary datum $h$ on the edge:

$$
c_{2}(y)=h(y, 0) / \cos \omega(y) .
$$

Then $c_{2} \in H_{\text {loc }}^{s-1}\left(M \backslash\left\{y_{0}, y_{0}^{\prime}\right\}\right)$ and $c_{2}$ generally blows up at $y_{0}$ and $y_{0}^{\prime}$.

In order to get the representation of $u_{\text {sing }}$ according to Theorem 3.1 at the crossing points, we only need again the imaginary parts of two basis functions, for instance:

$$
S\left[\nu_{1}(y) ; \zeta\right]=\zeta^{\nu_{1}(y)}, \quad S\left[\nu_{1}(y), \nu_{2}(y) ; \zeta\right]=\frac{\zeta-\zeta^{\nu_{1}(y)}}{1-\nu_{1}(y)} .
$$

Then the asymptotics of $u$ can be written

$$
d_{1} * \Phi \operatorname{Im} S\left[\nu_{1} ; \zeta\right]+d_{2} * \Phi \operatorname{Im} S\left[\nu_{1}, \nu_{2} ; \zeta\right] .
$$

We also could have chosen $S\left[\nu_{1}(y), \nu_{2}(y) ; \zeta\right]$ and $S\left[\nu_{2}(y) ; \zeta\right]$ as basis functions.

As $\omega \rightarrow \pi / 2, \operatorname{Im} S\left[\nu_{1}(y), \nu_{2}(y) ; \zeta\right]$ tends to the logarithmic singularity $\operatorname{Im} \zeta \log \zeta$.

Now we can compare the two above representations (3.10) and (3.11) of a singular part. The following relations between the coefficients hold:

$$
\begin{array}{ll}
d_{1}=c_{1}+c_{2}, & c_{1}=d_{1}-d_{2} /\left(1-\nu_{1}\right) \\
d_{2}=c_{2}\left(1-\nu_{1}\right), & c_{2}=d_{2} /\left(1-\nu_{1}\right) .
\end{array}
$$

3.d. Aims for the future. It would be interesting to know whether asymptotics for general elliptic equations and systems can be described by similar formulas, i.e. by divided differences of some analytic generating functions.

For general second order elliptic equations we have obtained asymptotics with "geometric terms" $S_{\alpha}$ in the form

$$
\psi(y, \theta) S\left[\nu_{\kappa_{1}}(y), \ldots, \nu_{\kappa_{q}}(y) ; r\right]
$$

with analytic $\psi(y, \theta)$. Such formulas rely upon the fact that there exist analytic choices for the exponents. But such an analytic choice is generically impossible for fourth order operators such as the bilaplacian. The basic problem is the expression of the roots of a polynomial whose coefficients depend analytically on a parameter. The roots are algebraic but, in general, non-analytic functions of the parameter. Such situations of bifurcations are studied in [14]. In the general case there appear combinations of both crossings and bifurcations. We think that even then it will be possible to reach the aims we described at the end of the first section, i.e., to separate all that can be separated. 
N ot e. The detailed proofs of the results presented in this paper can be found in the preprint $[2]$.

\section{References}

[1] M. Costabel and M. Dauge, Edge asymptotics on a skew cylinder, in: International Workshop "Analysis in Domains and on Manifolds with Singularities", Breitenbrunn 1990, Teubner, Leipzig 1991, to appear.

[2] —, - , General edge asymptotics of solutions of second order elliptic boundary value problems II, Publications de Laboratoire d'Analyse Numérique R91017, Université de Paris VI, 1991.

[3] M. Dauge, Elliptic Boundary Value Problems in Corner Domains-Smoothness and Asymptotics of Solutions, Lecture Notes in Math. 1341, Springer, Berlin 1988.

[4] P. Grisvard, Boundary Value Problems in Non-Smooth Domains, Pitman, London 1985.

[5] V. A. Kondrat'ev, Boundary-value problems for elliptic equations in domains with conical or angular points, Trans. Moscow Math. Soc. 16 (1967), 227-313.

[6] - Singularities of a solution of Dirichlet's problem for a second order elliptic equation in a neighborhood of an edge, Differential Equations 13 (1970), 1411-1415.

[7] R. S. Lehman, Developments at an analytic corner of solutions of elliptic partial differential equations, J. Math. Mech. 8 (1959), 727-760.

[8] V. G. Maz'ya and B. A. Plamenevskiú, $L^{p}$ estimates of solutions of elliptic boundary value problems in a domain with edges, Trans. Moscow Math. Soc. 1 (1980), 49-97.

[9] —, - Estimates in $L^{p}$ and in Hölder classes and the Miranda-Agmon maximum principle for solutions of elliptic boundary value problems in domains with singular points on the boundary, Amer. Math. Soc. Transl. (2) 123 (1984), 1-56.

[10] V. G. Maz'ya und J. Rossmann, Über die Asymptotik der Lösungen elliptischer Randwertaufgaben in der Umgebung von Kanten, Math. Nachr. 138 (1988), 27-53.

[11] _-, - On a problem of Babuška (Stable asymptotics of the solution of the Dirichlet problem for elliptic equations of second order in domains with angular points), preprint LiTH-MATR-90-33, Linköping University, 1991.

[12] V. A. Nikishkin, Singularities of the solution of the Dirichlet problem for a second order equation in a neighborhood of an edge, Moscow Univ. Math. Bull. 34 (2) (1979), 53-64.

[13] S. Rempel and B. W. Schulze, Asymptotics for Elliptic Mixed Boundary Problems, Akademie-Verlag, Berlin 1989.

[14] B. Schmutzler, The structure of branching asymptotics for elliptic boundary value problems in domains with edges, in: International Workshop "Analysis in Domains and on Manifolds with Singularities", Breitenbrunn 1990, Teubner, Leipzig 1991, to appear. 